\title{
Magnetohydrodynamic model of equatorial plasma torus in planetary nebulae
}

\author{
K. H. Tsui \\ Instituto de Física - Universidade Federal Fluminense Campus da Praia Vermelha, Av. General Milton Tavares de Souza s/n Gragoatá, \\ 24.210-346, Niterói, Rio de Janeiro, Brasil \\ e-mail: tsui@if.uff.br
}

Received 9 November 2007 / Accepted 29 January 2008

\begin{abstract}
Aims. Some basic structures in planetary nebulae are modeled as self-organized magnetohydrodynamic (MHD) plasma configurations with radial flow.

Methods. These configurations are described by time self-similar dynamics, where space and time dependences of each physical variable are in separable form. Axisymmetric toroidal MHD plasma configuration is solved under the gravitational field of a central star of mass $M$.

Results. With an azimuthal magnetic field, this self-similar MHD model provides an equatorial structure in the form of an axisymmetric torus with nested and closed toroidal magnetic field lines. In the absence of an azimuthal magnetic field, this formulation models the basic features of bipolar planetary nebulae. The evolution function, which accounts for the time evolution of the system, has a bounded and an unbounded evolution track governed respectively by a negative and positive energy density constant $H$.
\end{abstract}

Key words. magnetohydrodynamics (MHD) - methods: analytical - stars: AGB and post-AGB - ISM: planetary nebulae: general

\section{Introduction}

Planetary nebulae are believed to be intermediate-mass stars that are expelling their outer layers, at slow velocity, during the asymptotic giant branch (AGB) phase. As the temperature of the star increases, the ejected material becomes tenuous but moves with increasing speed. The hydrodynamic interaction of these slow and fast winds, at the intersection of their trajectories, generates shock waves that create the characteristic morphologies of planetary nelulae (Dyson \& de Vries 1972; Kwok et al. 1978). The outward-shock compresses and heats the slow dense wind that is ahead, and the inward-shock decelerates and heats the fast wind behind. This interacting-wind model reproduces the spherical features of planetary nebulae. In contrast, the elliptical and bipolar features may be created by a dense toroidal cloud in the equatorial plane (Kahn \& West 1985; Mellema et al. 1991). Although toroidal clouds are imaged by high-resolution instruments, how such structures formed during the AGB phase is still a debated issue. Besides spherical, elliptical, and bipolar features, high-resolution images have revealed point-symmetric features (Miranda \& Solf 1992; Lopez et al. 1993; Balick et al. 1993). These point-symmetric features, plus the detection of significant magnetic fields inside central stars (Jordan et al. 2006), appear to call for a magnetohydrodynamic (MHD) approach to the study of planetary nebulae (Pascoli 1993; Chevalier \& Luo 1994; Garcia-Segura 1997; Bogovalov \& Tsinganos 1999; Matt et al. 2000; Gardiner \& Frank 2001). In this paper, we present such an MHD analysis, which is able to recreate axisymmetric structures such as an equatorial plasma torus and a bipolar nebula. We consider an MHD plasma that simulates a radial-flow explosion in spherical coordinates, and we solve for solutions that are self-similar in time (Sommerfeld 1950; Landau \& Lifshitz 1978).
Self-similar MHD analysis was pioneered by Low (1982a,b, 1984a,b), in astrophysical stellar-envelope applications. The association of self-similar solutions to eruptive phenomena can be best demonstrated by the generation of self-similar shocks in powerful atmospheric detonations. The shocks are the results of self-organization, through dissipative processes, following chaos. The shock is built up as the gas expands outward, and it takes its fully developed form beyond a certain radius. These ordered structures could have a scale invariance that lowers the dimensionality of the time-dependent fluid system, and could exist between one spatial coordinate and time. This invariance is identified as the self-similar parameter, and similarity is temporal. Time evolution would be a separable factor, and, as a result, the time and space dependencies of self-similar variables are represented in separable form. For steady-state systems, the scale invariance could be between one spatial coordinate and another, and the similarity is said to be spatial.

Self-similarity is often regarded as a method to derive specific types of time-dependent or steady-state solutions. In this paper, we consider self-similarity to be a property of selforganized states, at least some of which are derived from turbulence. It is essential to note that the appearance of self-organized states, in fluids and magnetofluids, owes itself to the existence of multiple quadratic invariants in the absence of dissipations (Hasegawa 1985; Biskamp 1993). In the case of MHD fluids, the invariants are the total energy density (magnetic, plasma, and thermal), magnetic helicity, and in the case of incompressible fluids, cross-helicity. In the presence of dissipation, these invariants could undergo constrained changes, for example, from an MHD configuration, permitted by the MHD equations, to another topologically accessible configuration of lower energy. It is important to point out that these configurations are isolated in ideal cases. The continuous topological transformation from 
one to another configuration is only possible through dissipative paths such as magnetic reconnections. Mathematically speaking, this evolution amounts to the application of the variational principle, on the total energy density, under the constraint of global magnetic helicity conservation (Hasegawa 1985). The exact processes of dissipation, in taking the system to self-organization, need not be specified under the variational description. For this reason, MHD systems have the tendency to develop selforganized states with structural stability.

Since the self-similar method solves for the end configurations, directly from the governing equations, there is no need to specify the initial conditions. The plasma would find its way through magnetic reconnection, and other dissipative means, to reach one of the possible end configurations. The only condition is that the initial configuration is dissipatively transformable to the final configuration. If the initial conditions are close to those of the end configuration, the time to reach the end configuration is brief. Naturally, there are many end configurations, and only one of them will be selected by dissipative reorganization. For this reason, we cannot predict which self-similar state the system will reach. We can only match a system to a given self-similar solution that bears some resemblance.

One of the fundamental obstacles to visualizing self-similar solutions is an attempt to associate a given initial configuration with a specific self-similar end solution. It is possible to predict the final state of ideal MHD systems, in particular, by iterating equations of motion for an initial configuration. In the presence of dissipations, it can, however, be difficult to predict the final state, because of intermediate, chaotic events that occur while states are being reorganized. The initial configuration could undergo topological changes, through dissipative processes, such as magnetic reconnections, subject to the constraint of quadratic invariants. To describe the self-organized states arising from chaos, we have to override the forward time iteration process and attempt to identify the end configurations directly from the governing equations of the system. Although these end configurations have originated from dissipative reorganization through chaos, it is important to emphasize that these end configurations are determined by the ideal governing equations. Ideal MHD cannot, however, determine the dissipative path taken to reach one configuration from another. As a consequence, we are unable to recover the initial conditions by following the self-similar configurations backward in time. The initial conditions are lost from memory.

Recently, time self-similar analysis was applied to represent extragalactic jets as a polar-launched ejection event (Tsui \& Serbeto 2007), which differs from the classical accretionejection space self-similar steady state MHD transport model (Blandford \& Payne 1982). A self-similar description has been applied to different problems in physics, such as atmospheric ball lightnings (Tsui 2006; Tsui et al. 2006), and interplanetary magnetic ropes (Osherovich et al. 1993, 1995; Tsui \& Tavares 2005). Here, we follow the methods of time self-similar MHD analysis to model plasma torus, by developing solutions that are launched onto the equatorial plane.

\section{Axisymmetric self-similar MHD scalings}

The basic MHD equations, in Eulerian fluid description, are given by

$$
\frac{\partial \rho}{\partial t}+\nabla \cdot(\rho \boldsymbol{v})=0
$$

$\rho\left\{\frac{\partial \boldsymbol{v}}{\partial t}+(\boldsymbol{v} \cdot \nabla) \boldsymbol{v}\right\}=\boldsymbol{J} \times \boldsymbol{B}-\nabla p-\rho \frac{G M}{r^{3}} \boldsymbol{r}$,

$\frac{\partial \boldsymbol{B}}{\partial t}=-\nabla \times \boldsymbol{E}=\nabla \times(\boldsymbol{v} \times \boldsymbol{B})$,

$\nabla \times \boldsymbol{B}=\mu \boldsymbol{J}$,

$\nabla \cdot \boldsymbol{B}=0$

$\frac{\partial}{\partial t}\left(\frac{p}{\rho^{\gamma}}\right)+(\boldsymbol{v} \cdot \nabla)\left(\frac{p}{\rho^{\gamma}}\right)=0$,

where $\rho$ is the mass density, $\boldsymbol{v}$ is the bulk velocity, $\boldsymbol{J}$ is the current density, $\boldsymbol{B}$ is the magnetic field, $p$ is the plasma pressure, $\mu$ is the free space permeability, $\gamma$ is the polytropic index, and $M$ is the central mass, which provides the gravitational field.

We consider a radially expanding plasma, and look for selfsimilar solutions in time, where time evolution is described by the dimensionless evolution function $y(t)$. For this purpose, it is most convenient to think of Lagrangian fluid description, and consider the position vector of a given laminar flow fluid element $\boldsymbol{r}(t)$. Under self-similarity, the radial profile is time invariant in terms of the radial label $\eta=r(t) / y(t)$, which has the dimension of $r$. Physically, $\eta$ is the Lagrangian radial position of a fixed fluid element. With a finite plasma, the domain of $\eta$ is bounded by mass conservation

$0<\eta_{\text {int }}<\eta<\eta_{\text {ext }}$.

As for the plasma velocity, we consider self-similar structures deriving from a spherically symmetric radial velocity that can be written as

$\boldsymbol{v}=\frac{\mathrm{d} \boldsymbol{r}(t)}{\mathrm{d} t}=\left\{\eta \frac{\mathrm{d} y}{\mathrm{~d} t}, 0,0\right\}=\{v, 0,0\}$.

Our self-similar parameter $\eta$, defined through the Lagrangian fluid label, explicitly represents the fluid velocity by the time evolution function $y(t)$. This evolution function will be solved self-consistently with respect to the spatial structures of the plasma. We emphasize that self-similarity, as a method, can be applied in different ways other than the one we use here. For example, Lou and his collaborators have treated an aggregating fluid under its self-gravitational field with a similarity variable $x=r(t) / a t$, where $a$ is the sound speed, for isothermal fluid, $\gamma=1$, (Lou \& Shen 2004; Bian \& Lou 2005) and for a polytropic gas, $\gamma>1$, (Lou \& Wang 2006; Lou \& Gao 2006) to study relevant astrophysical phenomena. Extensions to a magnetofluid have been considered by Yu \& Lou (2005) and by Lou \& Wang (2007). Because of the linear dependence on time, this similarity variable $x$ refers to a reference frame moving at speed $a$, which is different from the radial plasma flow velocity $v$. Furthermore, different from our similarity variable $\eta, x$ here is not the Lagrangian label of a given fluid element. For this reason, the convective derivative remains explicit in the $x$ representation. As a result, the similarity variable of Lou amounts to finding the plasma structures in an adequate moving frame in the Eulerian $x$ fluid description. This resembles the analytic technique of going to a moving frame to look for stationary profile solutions for nonlinear phenomena such as nonlinear Alfven waves, solitons, etc. Because of this fundamentally different definition of self-similarity, the nature of the phenomena intended to describe is different. Our Lagrangian label self-similarity parameter is aimed to find spatial plasma configurations, and to determine the radial plasma flow velocity, consistent to the spatial configurations, through the evolution function. 
Since we are considering an isotropic radial plasma flow, a natural solution would be a hydrodynamic one-dimensional expanding plasma, with radially dependent mass density $\rho$ and plasma pressure $p$, and with $\boldsymbol{B}=0$ and $\boldsymbol{J}=0$. Nevertheless, this one-dimensional hydrodynamic solution is highly unlikely because magnetic field fluctuations can be generated from current density fluctuations, even in the absence of a pre-existing magnetic field. With the magnetic fields, which are basically a two- or three-dimensional structure, coupling to the plasma will generate likewise two- or three-dimensional $\rho$ and $p$. To examine the possible spatial structures of MHD plasmas, we consider a two-dimensional case with azimuthal symmetry in $\phi$. In this case, the magnetic field, through the vector potential $\boldsymbol{A}$, can be expressed as

$$
\begin{aligned}
\boldsymbol{B}= & \frac{1}{r \sin \theta}\left\{+\frac{1}{r} \frac{\partial}{\partial \theta}\left(r A_{\phi} \sin \theta\right),-\frac{\partial}{\partial r}\left(r A_{\phi} \sin \theta\right),\right. \\
& \left.+\sin \theta\left[\frac{\partial}{\partial r}\left(r A_{\theta}\right)-\frac{\partial}{\partial \theta}\left(A_{r}\right)\right]\right\} .
\end{aligned}
$$

This enables the magnetic field, the current density, and the electric field, to be expressed as two scalar functions $P$ and $Q$ respectively

$$
\begin{aligned}
\boldsymbol{B}= & \frac{1}{r \sin \theta}\left\{+\frac{1}{r} \frac{\partial}{\partial \theta}(P),-\frac{\partial}{\partial r}(P),+Q\right\} \\
= & \nabla P \times \nabla \phi+Q \nabla \phi, \\
\mu \boldsymbol{J}= & \frac{1}{r \sin \theta}\left\{+\frac{1}{r} \frac{\partial Q}{\partial \theta},-\frac{\partial Q}{\partial r},\right. \\
& \left.-\frac{\partial^{2} P}{\partial r^{2}}-\frac{1}{r^{2}} \sin \theta \frac{\partial}{\partial \theta}\left(\frac{1}{\sin \theta} \frac{\partial P}{\partial \theta}\right)\right\}, \\
\boldsymbol{E}= & -(\boldsymbol{v} \times \boldsymbol{B})=\frac{1}{r \sin \theta}\left\{0, v Q, v \frac{\partial P}{\partial r}\right\} .
\end{aligned}
$$

The independent variables are transformed from $(r, \theta, t)$ space to $(\eta, \theta, y)$ space. We determine the explicit dependence of $y$ on each one of the physical variables with this radial velocity using functional analysis. First, making use of Eq. (7), Eq. (1) renders

$$
\begin{aligned}
\frac{\partial \rho}{\partial t}+\frac{1}{r^{2}} \frac{\partial}{\partial r}\left(r^{2} v \rho\right) & =\left(\frac{\partial \rho}{\partial t}+v \frac{\partial \rho}{\partial r}\right)+\rho\left(\frac{\partial v}{\partial r}+\frac{2 v}{r}\right) \\
& =\frac{\partial \rho}{\partial y} \frac{\mathrm{d} y}{\mathrm{~d} t}+\frac{3 \rho}{y} \frac{\mathrm{d} y}{\mathrm{~d} t}=\left(\frac{\partial \rho}{\partial y}+\frac{3 \rho}{y}\right) \frac{\mathrm{d} y}{\mathrm{~d} t}=0 .
\end{aligned}
$$

To reach the second equality, we note that the first bracket, in the first equality, corresponds to the total time derivative of an Eulerian fluid element, which amounts to the time derivative of a Lagrangian fluid element. As for the second bracket, it can be reduced by using $v=\mathrm{d} r / \mathrm{d} t=\eta \mathrm{d} y / \mathrm{d} t$ and $\partial v / \partial r=(1 / y)(\mathrm{d} y / \mathrm{d} t)$. Solving this equation for $y$ scaling, by separating the time part, gives

$\rho(\boldsymbol{r}, t)=\frac{1}{y^{3}} \bar{\rho}(\eta, \theta)$.

As for Eq. (6), with $\alpha_{0} F=\left(p / \rho^{\gamma}\right)$ where $\alpha_{0}$ is a constant that carries the physical dimension so that $F$ is a dimensionless function, it follows

$\frac{\partial F}{\partial t}+v \frac{\partial F}{\partial r}=\frac{\partial F}{\partial y} \frac{\mathrm{d} y}{\mathrm{~d} t}=0$

$$
\left(\frac{p}{\rho^{\gamma}}\right)=\alpha_{0} F(\boldsymbol{r}, t)=\frac{1}{y^{0}} \alpha_{0} \bar{F}(\eta, \theta) .
$$

As for Eq. (3), using the representation of Eq. (8a), the magnetic functions $P$ and $Q$ are

$$
\begin{aligned}
& \frac{\partial P}{\partial t}+v \frac{\partial P}{\partial r}=\frac{\partial P}{\partial y} \frac{\mathrm{d} y}{\mathrm{~d} t}=0, \\
& P(\boldsymbol{r}, t)=\frac{1}{y^{0}} \bar{P}(\eta, \theta),
\end{aligned}
$$

$$
\begin{aligned}
& \frac{\partial Q}{\partial t}+\frac{\partial}{\partial r}(v Q)=\frac{\partial Q}{\partial y} \frac{\mathrm{d} y}{\mathrm{~d} t}+\frac{Q}{y} \frac{\mathrm{d} y}{\mathrm{~d} t} \\
&=\left(\frac{\partial Q}{\partial y}+\frac{Q}{y}\right) \frac{\mathrm{d} y}{\mathrm{~d} t}=0 \\
& Q(\boldsymbol{r}, t)=\frac{1}{y^{1}} \bar{Q}(\eta, \theta)
\end{aligned}
$$

\section{Self-similar formulation}

We have reduced the general set of time-dependent ideal MHD equations, Eqs. (1)-(6), to a set of self-similar equations with appropriate time scalings, Eqs. (7)-(12), and will seek solutions of Eq. (2) and the time evolution function $y(t)$. The general ideal MHD set of equations has nonlinear terms of the convective type $(\boldsymbol{v} \cdot \nabla)$ in Eqs. (1) and (2), and interaction type $(\boldsymbol{J} \times \boldsymbol{B})$ in Eq. (2) and $(\boldsymbol{v} \times \boldsymbol{B})$ in Eq. (3). By using the fluid label description, the $(\boldsymbol{v} \cdot \nabla)$ convective terms are absorbed into the Lagrangian time derivative representation. The $(\boldsymbol{v} \times \boldsymbol{B})$ interaction term is also absorbed, through an adequate representation of the magnetic field, by scalar functions $P$ and $Q$. The structure of the nonlinear terms, absorbed into the Lagrangian fluid label formulation, will appear in the $\eta$ profile of the system. The remaining task is to solve Eq. (2) for the self-similar $\eta$ structure, under the presence of the $(\boldsymbol{J} \times \boldsymbol{B})$ interaction term.

We note that the equation of $\alpha_{0} F=\left(p / \rho^{\gamma}\right)$ and the equation of $P$ are of the same form, and conclude that $F=F(P)$ is a functional of $P$ only, or $\bar{F}=\bar{F}(\bar{P})$. The fact that two equations have the same form does not automatically imply that the solution of one equation is a functional of the solution to the other. We can make this assertion only when considering self-similar solutions. We proceed to write the $(\eta, \theta)$ dependences in terms of $(\eta, \bar{P}(\eta, \theta))$ in $\bar{\rho}$ and $\bar{p}$, to obtain

$\bar{\rho}=\bar{\rho}(\eta, \bar{P})$,

$$
\begin{aligned}
p & =\frac{1}{y^{3 \gamma}} \alpha_{0} \bar{F}(\eta, \theta) \bar{\rho}^{\gamma}(\eta, \theta) \\
& =\frac{1}{y^{3 \gamma}} \alpha_{0} \bar{F}(\bar{P}) \bar{\rho}^{\gamma}(\eta, \bar{P})=\frac{1}{y^{3 \gamma}} \bar{p}(\eta, \bar{P}) .
\end{aligned}
$$

Furthermore, since $\left(p / \rho^{\gamma}\right)=\alpha_{0} F(P)$ is a function of $P$ only, the $\eta$ and $\bar{P}$ dependences should be in a separable form in both

$\bar{\rho}(\eta, \bar{P})=\rho_{0} \bar{\rho}_{1}(\eta) \bar{\rho}_{2}(\bar{P})$,

$\bar{p}(\eta, \bar{P})=p_{0} \bar{p}_{1}(\eta) \bar{p}_{2}(\bar{P})$.

The dimensions of mass density and pressure appear explicitly in $\rho_{0}$ and $p_{0}$ respectively. In this form, $\bar{\rho}_{1}(\eta), \bar{\rho}_{2}(\bar{P}), \bar{p}_{1}(\eta)$, and 
$\bar{p}_{2}(\bar{P})$ are dimensionless functions. By taking $\alpha_{0}=p_{0} / \rho_{0}^{\gamma}$ with the physical dimension, we have

$\bar{p}_{1}(\eta)=\bar{\rho}_{1}^{\gamma}(\eta)$

$\bar{p}_{2}(\bar{P})=\bar{F}(\bar{P}) \bar{\rho}_{2}^{\gamma}(\bar{P})$,

such that $\bar{F}$ should be a functional of $\bar{P}$ only.

Making use of Eq. (4) to eliminate the current density in Eq. (2), we derive the momentum equation, which has three components. The $\phi$ component, which contains only the magnetic force, gives

$\frac{\partial P}{\partial r} \frac{\partial Q}{\partial \theta}-\frac{\partial P}{\partial \theta} \frac{\partial Q}{\partial r}=\frac{1}{y^{2}}\left\{\frac{\partial \bar{P}}{\partial \eta} \frac{\partial \bar{Q}}{\partial \theta}-\frac{\partial \bar{P}}{\partial \theta} \frac{\partial \bar{Q}}{\partial \eta}\right\}=0$,

$\bar{Q}=\bar{Q}(\bar{P})$.

The function $P$ plus the functional form of $Q$, therefore, determines the magnetic fields. As for the $\theta$ component, with $p=\bar{p}(\eta, \bar{P}) / y^{3 \gamma}$, it reads

$$
\begin{array}{r}
\frac{\partial^{2} \bar{P}}{\partial \eta^{2}}+\frac{1}{\eta^{2}} \sin \theta \frac{\partial}{\partial \theta}\left(\frac{1}{\sin \theta} \frac{\partial \bar{P}}{\partial \theta}\right)+\bar{Q} \frac{\partial \bar{Q}}{\partial \bar{P}} \\
+\mu y^{4-3 \gamma} \eta^{2} \sin ^{2} \theta \frac{\partial \bar{p}}{\partial \bar{P}}=0 .
\end{array}
$$

We remark that the first three terms of this equation represent the nonlinear force-free field equation with $\bar{Q}=\bar{Q}(\bar{P})$. In the particular case of a linear functional $\bar{Q}=a \bar{P}$, this equation describes the linear force-free fields, which can be verified easily from Eqs. (8) with $\mu \boldsymbol{J}=a \boldsymbol{B}$. Such a force-free magnetic configuration is the marker of self-organized plasmas, and is the result of the variational principle that minimizes the energy density of the magnetic field, under the constraint of global magnetic helicity conservation. This justifies the use of self-similarity method to describe self-organized configurations. The last term of Eq. (17) is the plasma pressure term. For an isotropic pressure independent of $\bar{P}$, this last term would be null and such plasma pressure $\bar{p}(\eta)$ has no effect on the magnetic field which remains force-free. For an axisymmetric pressure $\bar{p}(\eta, \bar{P})$, the above equation would be independent of the time evolution function $y$ should $\gamma=4 / 3$, and it would describe a plasma pressure balanced axisymmetric magnetic field. Such a pressure-balanced self-organized configuration could be derived using the variational principle, by minimizing the energy density that contains not only magnetic energy but also plasma thermal energy under the same constraint. The solution of this equation of $P$, with different representations of $\bar{Q}=\bar{Q}(\bar{P})$ and $\bar{p}=\bar{p}(\bar{P})$, is the core problem of self-similar description.

As for the $r$ component of the momentum equation, it reads

$$
\begin{aligned}
\rho\left[\frac{\partial v}{\partial t}+v \frac{\partial v}{\partial r}\right] & +\frac{\mathrm{d}}{\mathrm{d} r} p(r, P(r, \theta))+\rho \frac{G M}{r^{2}}= \\
- & \frac{1}{\mu}\left(\frac{1}{r \sin \theta}\right)^{2} \frac{\partial P}{\partial r}\left\{\frac{\partial^{2} P}{\partial r^{2}}\right. \\
& \left.+\frac{1}{r^{2}} \sin \theta \frac{\partial}{\partial \theta}\left(\frac{1}{\sin \theta} \frac{\partial P}{\partial \theta}\right)+Q \frac{\partial Q}{\partial P}\right\} \\
= & \frac{\partial P}{\partial r} \frac{\partial}{\partial P} p(r, P) .
\end{aligned}
$$

The term $\mathrm{d} p / \mathrm{d} r$ on the left side refers to the total radial derivative of the plasma pressure, which includes both an explicit and implicit dependence in $P$. The right side of the radial equation provides an expression for the magnetic force. Making use of the meridian component, Eq. (17), the right side is equal to the implicit part of the radial pressure gradient, which cancels the same term on the left side. This leaves only the explicit radial pressure gradient

$$
\begin{aligned}
\rho\left[\frac{\partial v}{\partial t}+v \frac{\partial v}{\partial r}\right]+ & \frac{\partial}{\partial r} p(r, P)+\rho \frac{G M}{r^{2}}= \\
\rho \frac{\mathrm{d}^{2} r}{\mathrm{~d} t^{2}}+\frac{\partial}{\partial r} p(r, P)+\rho \frac{G M}{r^{2}} & =0 .
\end{aligned}
$$

In terms of self-similar parameters, the radial equation reads

$y^{2} \frac{\mathrm{d}^{2} y}{\mathrm{~d} t^{2}}+\frac{1}{\eta}\left\{y^{4-3 \gamma} \frac{1}{\bar{\rho}} \frac{\partial \bar{p}}{\partial \eta}+\frac{G M}{\eta^{2}}\right\}=0$.

With $\alpha$ as the separation constant, $\alpha_{2}$ as a dimensionless constant, and $\gamma=4 / 3$, we derive

$$
\begin{aligned}
& 4 \alpha_{2} \frac{p_{0}}{\rho_{0}} \bar{\rho}_{1}^{1 / 3}(\eta)=\left(\frac{1}{2} \alpha \eta^{2}+\frac{G M}{\eta}\right) \\
&=\frac{\alpha}{2 \eta}\left(\eta^{3}+\frac{2 G M}{\alpha}\right)=\frac{\alpha}{2 \eta}\left(\eta^{3}+2 \eta_{*}^{3}\right), \\
& \bar{p}_{2}(\bar{P})=\alpha_{2} \bar{\rho}_{2}(\bar{P}),
\end{aligned}
$$

$y^{2} \frac{\mathrm{d}^{2} y}{\mathrm{~d} t^{2}}=-\alpha$

The first of these three equations provides the mass density profile of the radial label. The second one specifies the $\bar{P}$ dependence between mass density and plasma pressure. The third one defines the time history of the evolution function.

\section{Axisymmetric equatorial structures}

After describing our self-similar formulation, we solve Eqs. (14), (17), and (20), for axisymmetric plasma configurations. We begin with comparing Eqs. (14b) and (20b), and conclude that

$\bar{F}(\bar{P})=\alpha_{2} \bar{\rho}_{2}^{1-\gamma}(\bar{P})$.

The solution to Eq. (17), for the magnetic field function $\bar{P}$, depends on the functionals $\bar{Q}$ and $\bar{p}_{2}$. This is where different types of self-similar configurations can be produced. Although Low pioneered the use of self-similar MHD in astrophysics, he did not explore the full potential of the method. We remark that self-similar solutions are highly dependent on the functional representations of $\bar{Q}$ and $\bar{p}_{2}$. Under a given geometry, there are different self-similar solutions, with different characteristics to represent different self-organized plasmas, depending on how the magnetic fields and plasma parameters are modeled. To study configurations on and about the equatorial plane, we consider

$\bar{Q}=a \bar{P}$,

$\bar{p}_{2}(\bar{P})=(\bar{P}+\bar{C})>0$.

For example, if we assume a quadratic function to have

$\bar{p}_{2}(\bar{P})=\left(\bar{P}^{2}+\bar{C}\right)>0$,

we derive a polar ejection configuration that tends to collimate magnetic fields and plasma density, onto the polar axis, as 
plasma pressure builds up (Tsui \& Serbeto 2007). Using these functional representations in Eq. (17), we derive

$$
\begin{aligned}
\eta^{2} \frac{\partial^{2} \bar{P}}{\partial \eta^{2}}+ & \sin \theta \frac{\partial}{\partial \theta}\left(\frac{1}{\sin \theta} \frac{\partial \bar{P}}{\partial \theta}\right)+(a \eta)^{2} \bar{P}= \\
& -\mu p_{0} \eta^{4} \bar{p}_{1}(\eta) \sin ^{2} \theta=-\mu p_{0} \eta^{4} \bar{\rho}_{1}^{4 / 3}(\eta) \sin ^{2} \theta
\end{aligned}
$$

To solve this equation, we separate the variables by writing $\bar{P}(\eta, \theta)=R(\eta) \Theta(\theta)$. Assuming $x=\cos \theta$ and $n(n+1)$ to be the separation constant, we have

$\left(1-x^{2}\right) \frac{\mathrm{d}^{2} \Theta(x)}{\mathrm{d} x^{2}}+n(n+1) \Theta(x)=0$,

$\eta^{2} \frac{\mathrm{d}^{2} R}{\mathrm{~d} \eta^{2}}+\left[(a \eta)^{2}-n(n+1)\right] R=-\mu p_{0} \eta^{4} \bar{\rho}_{1}^{4 / 3}(\eta) \frac{\sin ^{2} \theta}{\Theta(x)}$.

The first equation provides the well-known solution, in terms of the Legendre polynomial $P_{n}(x)$,

$\Theta(x)=C_{0}\left(1-x^{2}\right) \frac{\mathrm{d} P_{n}(x)}{\mathrm{d} x}=-n(n+1) C_{0} \int_{1}^{x} P_{n}(x) \mathrm{d} x$.

We note that the second equation is not in a separable form. Nevertheless, should we choose $n=1$ with $P_{1}(x)=x$, such that $\Theta(x)=\left(1-x^{2}\right)$, where we assume that $C_{0}=1$, Eq. (24b) is separable. The general solution is given by a homogeneous solution $R_{0}(\eta)$ and a particular solution $R_{1}(\eta)$

$R(\eta)=R_{0}(\eta)+R_{1}(\eta)=A_{0}(a \eta) j_{n}(a \eta)+R_{1}(\eta)$,

where $j_{n}(a \eta)$ with $n=1$ is the spherical Bessel function regular at $\eta=0$.

The coefficient $A_{0}$ of the homogeneous solution carries the amplitude and dimension to reflect the magnetic field through Eq. (8a). Since plasma pressure and mass density are expressed in terms of $P$ via $\bar{p}_{2}(\bar{P})$ and $\bar{\rho}_{2}(\bar{P})$, we could normalize the entire self-similar solution, with respect to the magnetic field, by taking $A_{0}=1$. With $\bar{\rho}_{1}(\eta)$ given by Eq. (20a), denoting $z=a \eta$, and $\kappa^{3}=2\left(a \eta_{*}\right)^{3}=2 z_{*}^{3}$, the particular solution is described by

$$
\begin{aligned}
z^{2} \frac{\mathrm{d}^{2} R_{1}}{\mathrm{~d} z^{2}}+\left[z^{2}-n(n+1)\right] R_{1} & =-\mu p_{0}\left(\frac{\alpha}{a^{3}}\right)^{4}\left(\frac{1}{8 \alpha_{2}} \frac{\rho_{0}}{p_{0}}\right)^{4}\left(z^{3}+\kappa^{3}\right)^{4} \\
& =-A\left(z^{3}+\kappa^{3}\right)^{4} .
\end{aligned}
$$

We note that the coefficient $A$, which has two factors, is dimensionless. The first factor $\left(\mu p_{0} / a^{4}\right) \simeq \beta / z_{*}^{2}$ is related to the plasma $\beta$, which is the ratio of plasma pressure to magnetic pressure. The second dimensionless factor with $G M$ is basically the ratio of the gravitational potential energy density to the plasma pressure. The right side of this equation can be expanded into binomial terms, and can be solved using power series, by taking one term at a time. The particular solution is, therefore, a superposition of five sub-solutions $R_{1(12)}, R_{1(9)}, R_{1(6)}, R_{1(3)}$, and $R_{1(0)}$, where the bracketed number in the subscript denotes the power of $z$ in the binomial term, on the right side. We denote the coefficients $A^{(12)}=A, A^{(9)}=4 \kappa^{3} A, A^{(6)}=6 \kappa^{6} A, A^{(3)}=4 \kappa^{9} A$, $A^{(0)}=\kappa^{12} A$. We solve each binomial term of the particular solution using a power series of the form

$R_{1(k)}(\eta)=\sum a_{m} z^{+m}$

Using standard power-series techniques, we derive solutions of the form

$R_{1(12)}(z)=a_{2} z^{2}+a_{4} z^{4}+a_{6} z^{6}+a_{8} z^{8}+a_{10} z^{10}$, where $a_{10}=-A^{(12)}, a_{8}=-(10 \times 9-2) a_{10}, a_{6}=-(8 \times 7-2) a_{8}$, $a_{4}=-(6 \times 5-2) a_{6}, a_{2}=-(4 \times 3-2) a_{4}$, and $a_{0}=-(2 \times 1-2) a_{2}=0$,

$R_{1(9)}(z)=a_{9} z^{9}+a_{11} z^{11}+a_{13} z^{13}+a_{15} z^{15}+\ldots \ldots$,

where $a_{9}=-A^{(9)} /(9 \times 8-2), a_{11}=-a_{9} /(11 \times 10-2)$, $a_{13}=-a_{11} /(13 \times 12-2), a_{15}=-a_{13} /(15 \times 14-2)$, $a_{m}=-a_{m-2} /(m \times(m-1)-2)$,

$R_{1(6)}(z)=a_{2} z^{2}+a_{4} z^{4}$

where $a_{4}=-A^{(6)}, a_{2}=-(4 \times 3-2) a_{4}$, and $a_{0}=-(2 \times 1-2) a_{2}$ $=0$,

$R_{1(3)}(z)=a_{3} z^{3}+a_{5} z^{5}+a_{7} z^{7}+a_{9} z^{9}+\ldots \ldots$

where $a_{3}=-A^{(3)} /(3 \times 3-2), a_{5}=-a_{3} /(5 \times 4-2), a_{7}=-a_{5} /(7 \times$ $6-2), a_{9}=-a_{7} /(9 \times 8-2), a_{m}=-a_{m-2} /(m \times(m-1)-2)$. As for $R_{1(0)}(z)$, this solution has to be solved with series of negative powers, which describes the divergent nature of the gravitational field $G M / \eta$ at $\eta=0$, although this limit is irrelevant here. We derive

$R_{1(0)}(\eta)=\sum a_{m} z^{-m}$

where $a_{0}=0, a_{2}=-A^{(0)}, a_{4}=-(2 \times 3-2) a_{2} /(4 \times 5), a_{6}=$ $-(4 \times 5-2) a_{4} /(6 \times 7), a_{8}=-(6 \times 7-2) a_{6} /(8 \times 9), a_{m}=$ $-((m-2) \times(m-1)-2) a_{m-2} /(m \times(m+1))$. We note that the homogeneous solution corresponds to force-free magnetic field configurations. Due to the plasma pressure in Eq. (17), there are particular solutions that maintain the pressure balance.

\section{Magnetic fields and evolution function}

After solving for the spatial structure of $\bar{P}$, the magnetic fields are given by

$$
\begin{aligned}
B_{r} & =\frac{1}{y^{2}} \bar{B}_{r}(\eta, \theta)=+\frac{1}{y^{2}} \frac{1}{\eta \sin \theta} \frac{1}{\eta} \frac{\partial \bar{P}}{\partial \theta} \\
& =-\frac{1}{y^{2}} \frac{1}{\eta^{2}} R(\eta) \frac{\mathrm{d} \Theta(x)}{\mathrm{d} x} \\
B_{\theta} & =\frac{1}{y^{2}} \bar{B}_{\theta}(\eta, \theta)=-\frac{1}{y^{2}} \frac{1}{\eta \sin \theta} \frac{\partial \bar{P}}{\partial \eta} \\
& =-\frac{1}{y^{2}} \frac{1}{\eta} \frac{\mathrm{d} R(\eta)}{\mathrm{d} \eta} \frac{1}{\left(1-x^{2}\right)^{1 / 2}} \Theta(x), \\
B_{\phi} & =\frac{1}{y^{2}} \bar{B}_{\phi}(\eta, \theta)=+\frac{1}{y^{2}} \frac{1}{\eta \sin \theta} \bar{Q} \\
& =+\frac{1}{y^{2}} \frac{a}{\eta} R(\eta) \frac{1}{\left(1-x^{2}\right)^{1 / 2}} \Theta(x) .
\end{aligned}
$$

The self-similar homogeneous solution $R_{0}(\eta)$, provided by Eq. (26), enables oscillations in $\eta$ to be modeled, and vanishes at the zeros of the spherical Bessel function. To understand the magnetic structures, we first set aside the particular solution $R_{1}(\eta)$. At the first zero of $R_{0}(\eta)$, we have $B_{\phi}(\eta)=0$, $B_{r}(\eta)=0$, and the only nonvanishing field is $B_{\theta}(\eta)$ according to Eqs. (28). The meridian self-similar solution $\Theta(x)$, given by Eq. (25), oscillates in $x$. Together they describe the magnetic fields of Eqs. (28). Within this region of $(\eta, x)$, the topological center, defined by $\mathrm{d} R_{0}(\eta) / \mathrm{d} \eta=0$ and $\mathrm{d} \Theta(x) / \mathrm{d} x=0$, has $B_{r}=0$, 
$B_{\theta}=0$, and the only nonvanishing field is $B_{\phi}(\eta)$. This is the magnetic axis. The field lines about the magnetic axis are, therefore, given by

$\frac{B_{r}}{\mathrm{~d} r}=\frac{B_{\theta}}{r \mathrm{~d} \theta}=\frac{B_{\phi}}{r \sin \theta \mathrm{d} \phi}$.

By axisymmetry, the third group is decoupled from the first two groups. For the field lines on an $(r-\theta)$ plane, we consider the first equality between $B_{r}$ and $B_{\theta}$, which gives

$\bar{P}(\eta, x)=R_{0}(\eta)\left(1-x^{2}\right)=C$.

The nested field lines are given by the contours of $\bar{P}(\eta, x)$ on the $(r-\theta)$ plane. At the topological center, the magnetic axis, we have $\Theta(x)$ at its maximum value, and $R_{0}(\eta)$ at its maximum, such that $\bar{P}(\eta, x)$ takes its maximum value. Since

$2 \pi r \sin \theta B_{\phi}=2 \pi a \bar{P}$,

by Eq. (28c), the line integral of $B_{\phi}$ on the magnetic axis is a maximum. We, therefore, have a sequence of plasma tori, due to the periodic nature of the spherical Bessel function. The presence of the particular solution $R_{1}(\eta)$ modifies this structure. Due to the divergent nature of $R_{1}(\eta)$, as we will see in the next section, only the first torus prevails.

As for the temporal part, the evolution function in Eq. (20c) is described by

$$
\begin{aligned}
& \frac{1}{2}\left(\frac{\mathrm{d} y}{\mathrm{~d} t}\right)^{2}=\left(H+\frac{\alpha}{y}\right)>0, \\
& \left(\frac{\mathrm{d} y}{\mathrm{~d} t}\right)=\left[2\left(H+\frac{\alpha}{y}\right)\right]^{1 / 2} .
\end{aligned}
$$

Here, $H$ is an integration constant that is independent of time. To understand the meaning of $\alpha$, we note that, using Eq. (20c), plasma acceleration in Lagrangian coordinates is

$$
\frac{\mathrm{d} v}{\mathrm{~d} t}=\eta \frac{\mathrm{d}^{2} y}{\mathrm{~d} t^{2}}=-\frac{\alpha \eta}{y^{2}} .
$$

A positive $\alpha$ corresponds to an outward, decelerating flow. The deceleration becomes smaller as $y$, or as $r$, becomes larger, and $\alpha$ equals the intensity of the deceleration, for a given radial label $\eta$. To derive an expression for $H$, we multiply Eq. (30a) by $\rho \eta^{2}$, and use Eq. (31a) to obtain

$$
\begin{aligned}
\rho \eta^{2} H & =\frac{1}{2} \rho v^{2}+\left(\rho \frac{\mathrm{d} v}{\mathrm{~d} t}\right) r \\
& =\frac{1}{2} \rho v^{2}-\left(\frac{\partial}{\partial r} p(r, P)+\rho \frac{G M}{r^{2}}\right) r .
\end{aligned}
$$

The first term on the right side is the kinetic energy density of the fluid element, and the second term is its work on expansion, due to the explicit part of the pressure gradient. We note that the magnetic energy does not appear in this expression because it is cancelled by the work due to the implicit pressure gradient, as discussed in Eq. (18a). It is clear that $H$ measures the total energy of the fluid element.

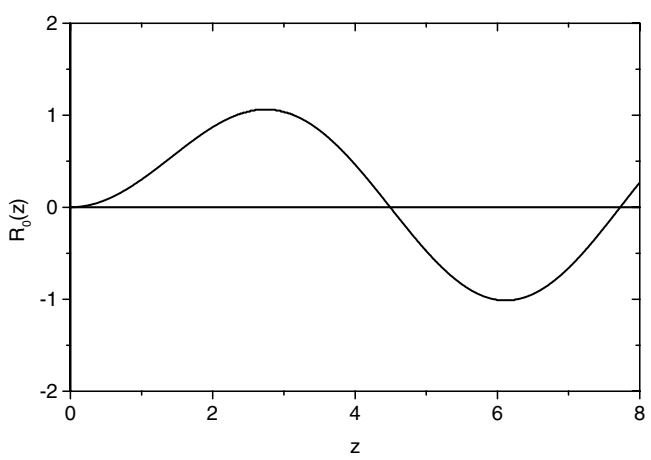

Fig. 1. The function $R_{0}(z)=A_{0} z j_{1}(z)$ of the homogeneous solution is plotted as a function of $z=a \eta$ to show the radial domains of the axisymmetric plasma structures.

\section{Force-free equatorial plasma torus}

We consider the explicit radial dependent part of the mass density, in Eq. (20a). Since $\bar{\rho}_{1}(\eta)$ is positive, $\alpha$ should also be positive. Differentiating with respect to $\eta$ indicates that $\bar{\rho}_{1}(\eta)$ has a minimum at

$\eta_{*}^{3}=\frac{G M}{\alpha}$.

Because we are considering a finite plasma, the range of the radial label $\eta$ is finite. We assume that $\eta_{*}$ is enclosed in the interval $0<\eta_{\text {int }}<\eta_{*}<\eta_{\text {ext }}$. For $\eta>\eta_{*}, \bar{\rho}_{1}(\eta)$ increases because of the $\alpha \eta^{2} / 2$ term, where $\alpha$ is defined by Eq. (20c) and measures the inertia of the plasma. A positive $\alpha$ corresponds to a decelerating plasma. Beyond the external bound is interstellar space, where plasma mass density decreases abruptly. In the presence of the gravitational term, $G M / \eta$, in Eq. (20a), the mass density $\bar{\rho}_{1}(\eta)$ rises again for $\eta<\eta_{*}$. This gravitational term is singular at $\eta=0$. However this singularity is not included, because the internal bound of $\eta$ is at the surface of the star.

To consider the function $\bar{P}(\eta, x)=R(\eta) \Phi(x)$, the homogeneous solution $R_{0}(\eta)=A_{0}(a \eta) j_{1}(a \eta)$, with $A_{0}=1$, is shown in Fig. 1, with the first node at $z=(a \eta)=4.5$. In the absence of plasma pressure, $R_{0}(\eta)$ would be the only term for $R(\eta)$. With $z_{\text {ext }}=a \eta_{\text {ext }}=4.5$, this would correspond to the equatorial plasma torus force-free magnetic fields. As for the particular solution $R_{1}(\eta)$, we note that $R_{1(12)}(z), R_{1(9)}(z), R_{1(6)}(z)$, and $R_{1(3)}(z)$ diverge as $z$ goes to infinity, whereas $R_{1(0)}(z)$ diverges as $z$ goes to zero. These two extremes are naturally excluded, because $z=0$ is the center of the central star, and $z=\infty$ requires an infinite plasma, which is unphysical. An adequate domain could be provided by the homogeneous solution plotted in Fig. 1, which shows an axisymmetric equatorial plasma torus with $0<z<4.5$, if the particular solution does not significantly alter this structure. The particular binomial solutions depend on the parameter $\kappa^{3}=2 G M a^{3} / \alpha=2\left(a \eta_{*}\right)^{3}$, where $a$ is a measure of the azimuthal magnetic field given by Eq. (22a). We assume the minimum $z_{*}=a \eta_{*}=2$, such that $\kappa^{3}=16$. We note that the two factors (brackets) in

$A=-\left(\frac{\mu p_{0}}{a^{4}}\right)\left(\frac{1}{8 \alpha_{2}} \frac{1}{z_{*}^{2}} \frac{\rho_{0} G M}{\eta_{*}} \frac{1}{p_{0}}\right)^{4}$

in the equation of $R_{1}(\eta)$ are dimensionless quantities. The first factor $\left(\mu p_{0} / a^{4}\right) \simeq \beta / z_{*}^{2} \simeq 10^{-1}$ can be expressed in terms of plasma $\beta$, which is the ratio of the plasma pressure to the magnetic pressure. This factor is estimated to be $10^{-1}$. The second factor is basically the ratio of the gravitational potential energy 


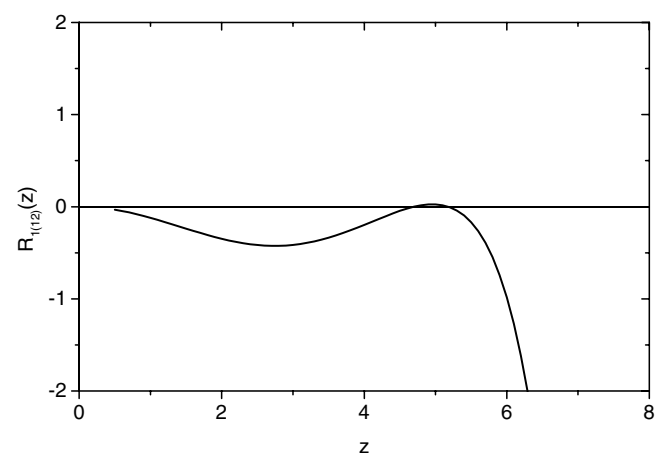

Fig. 2. The power series solution $R_{1(12)}(z)$ of the particular solution, with $A=1 \times 10^{-7}$, is plotted as a function of $z$ to show the plasma pressure effect on the radial domains of the axisymmetric plasma structures.

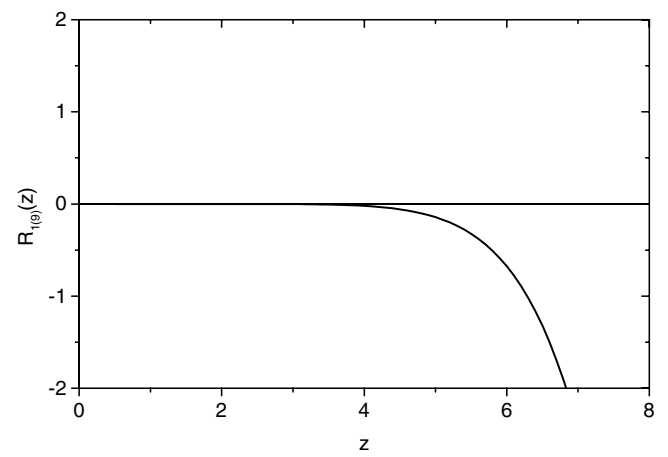

Fig. 3. The power series solution $R_{1(9)}(z)$ of the particular solution, with $A=1 \times 10^{-7}$ and $\kappa^{3}=16$, is plotted as a function of $z$ to show the plasma pressure effect on the radial domains of the axisymmetric plasma structures.

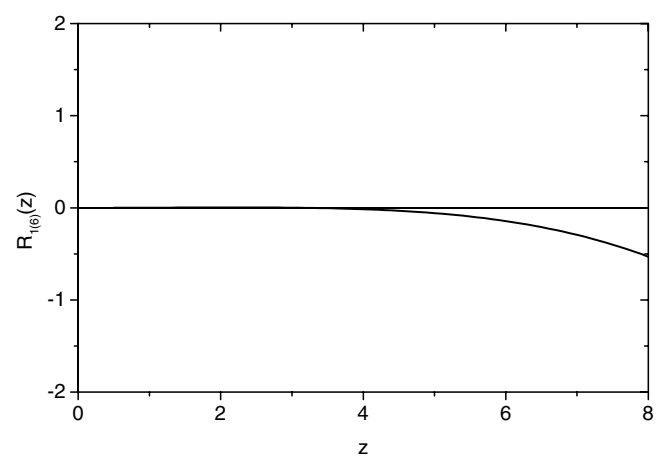

Fig. 4. The power series solution $R_{1(6)}(z)$ of the particular solution, with $A=1 \times 10^{-7}$ and $\kappa^{3}=16$, is plotted as a function of $z$ to show the plasma pressure effect on the radial domains of the axisymmetric plasma structures.

density $\rho_{0} G M / \eta_{*}$ to the plasma pressure $p_{0}$, plus other dimensionless multipliers to the fourth power. We believe that the gravitational potential energy density and the plasma pressure are approximately similar. We could take $\alpha_{2}=1$, and the remaining terms would provide the factor $10^{-6}$. Multiplying the two factors together gives $A=10^{-7}$.

Of the five parts of the particular solution $R_{1}, R_{1(12)}$ is unaffected by the choice of $\kappa^{3}$, and is plotted in Fig. 2. Other binomial parts $R_{1(9)}, R_{1(6)}, R_{1(3)}$, plotted in Figs. 3-5 respectively, carry mixed contributions of plasma pressure and gravitational field, and are negligible within the domain. The last part $R_{1(0)}$ in Fig. 6 is purely gravitational. The neighborhood of the center of the star, $z=0$, is excluded. These binomial parts depend

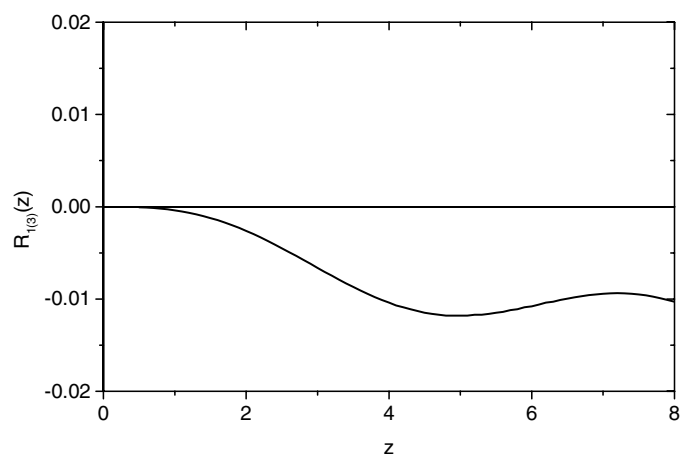

Fig. 5. The power series solution $R_{1(3)}(z)$ of the particular solution, with $A=1 \times 10^{-7}$ and $\kappa^{3}=16$, is plotted as a function of $z$ to show the plasma pressure effect on the radial domains of the axisymmetric plasma structures.

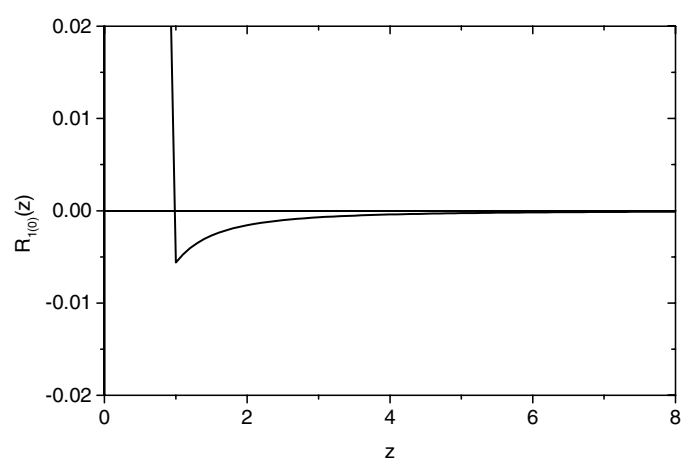

Fig. 6. The power series solution $R_{1(0)}(z)$ of the particular solution, with $A=1 \times 10^{-7}$ and $\kappa^{3}=16$, is plotted as a function of $z$ to show the plasma pressure effect on the radial domains of the axisymmetric plasma structures.

on the choice of $\kappa^{3}$, but would have a negligible effect on $R_{0}$, within the domain of interest. The homogeneous and the particular solutions provide the complete solution for $R(\eta)$. Together with $\Theta(x)$, they determine the function $\bar{P}(\eta, x)$, which governs the entire axisymmetric self-similar profile in magnetic fields, plasma pressure, and mass density.

To consider the magnetic field profiles, we recall that the field lines on an $(r-\theta)$ plane are given by Eq. (29a), with $R(z)=R_{0}(z)+R_{1}(z)$. The presence of a plasma gives a diamagnetic effect, as shown by $R_{1(12)}(z)$ in Fig. 2, carrying an opposite sign with respect to $R_{0}(z)$ of Fig. 1. Examining Fig. 2, we note that $R_{1(12)}$ vanishes at $z=4.5$ and $z=5.2$. The first node of $R_{1(12)}$ happens to be at the same location as the first node of $R_{0}$, also at $z=4.5$. Furthermore, Figs. $3-6$ show that $R_{1(9)}$, $R_{1(6)}, R_{1(3)}, R_{1(0)}$ are all negligibly small. Consequently, the general solution $R(z)=R_{0}(z)+R_{1}(z)=0$ at $z=4.5$. By Eqs. (28), we conclude that the radial and azimuthal magnetic fields, $B_{r}(z)$ and $B_{\phi}(z)$, vanish at this location, while the meridian magnetic field, $B_{\theta}(z)$, is maximum. Consequently, the domain $0<z<4.5$ constitutes a region for a magnetic torus with closed field lines. Since plasma is tied to the magnetic field lines, it circulates along and is confined within this torus. We note that the sign of $R_{1}$ is negative, which indicates the diamagnetic nature of plasma. We note that $R_{1(12)}$ has a maximum at the same location as $R_{0}$. As a matter of fact, the functional form of $R_{1(12)}$ in the interval $(0,4.5)$ is much the same as that of the force-free $R_{0}$. Consequently, the general solution $R$ is practically force-free, even when plasma pressure is taken into account, in Eq. (23). 


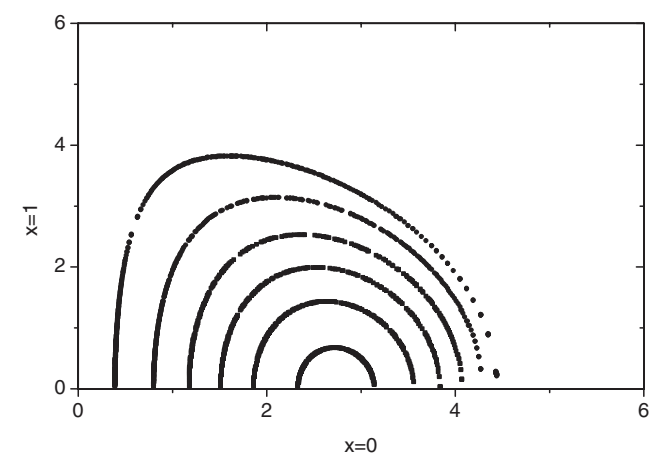

Fig. 7. The magnetic field lines using the homogeneous solution $R_{0}(z)$ are shown with $C=+0.05, C=+0.20, C=+0.40, C=+0.60, C=$ +0.80 , and $C=+1.00$ from the outer to inner contours respectively. The axes are labelled in $x=\cos \theta$, and the contribution of the plasma pressure represented by $R_{1}(z)$ is neglected here.

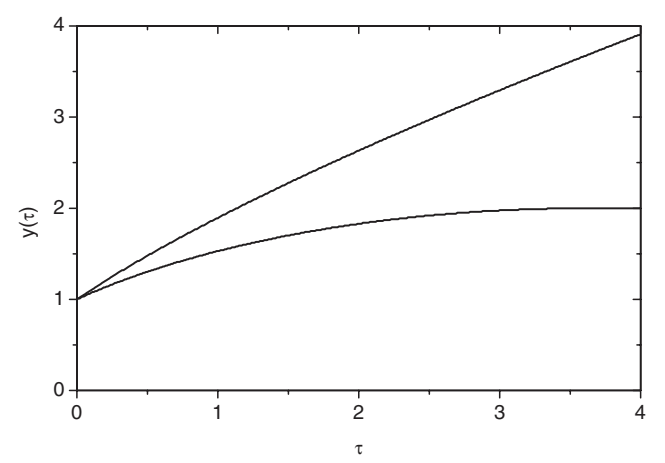

Fig. 8. The asymptotically bounded evolution function, the lower curve, is plotted as a function of the normalized time $\tau$ with $|H| / \alpha=0.5$, whereas the unbounded evolution function, the upper curve, is plotted with $H / \alpha=0.1$.

For simplicity, we neglect the diamagnetic effect, and take $R(z)=R_{0}(z)$ to highlight the poloidal field lines in Fig. 7, which is described by first equality of the field line equation, with solutions in Eq. (29a). The addition of $R_{1(12)}(z)$ would change the contours slightly, but not the topology. Apart from these poloidal fields, there are associated toroidal fields given by Eq. (28c). The maxima of $R$ and $\Theta, \partial \bar{P} / \partial \eta=0$ and $\partial \bar{P} / \partial \theta=0$, give the maximum of this toroidal field where $B_{r}=0$ and $B_{\theta}=0$ respectively. This corresponds to the magnetic axis of the plasma torus. Together, the field lines thread out nested magnetic surfaces enclosing the magnetic axis, one within another. The plasma pressure and mass density of this plasma torus have $R(z)>0$. With $\Theta(x)>0$, this warrants $\bar{P}(z, x)>0$ to assure positive pressure and mass density. With $\bar{C}=0$ in Eq. (22b), the plasma pressure and mass density vanish at $z=4.5$ since $R(z)=0$. It is important to note that, for $z>4.5$, the particular solution $R_{1}(z)$ dominates the homogeneous solution $R_{0}(z)$ such that the general solution $R(z)$ never vanishes. The radial component of the magnetic field is nonzero. For this reason, the field lines are open to outer space, and the plasma is no longer confined. This outer open plasma structure is not shown in Fig. 7.

For the evolution function, it is most convenient to write Eq. (30b) in the normalized form

$$
\left(\frac{\mathrm{d} y}{\mathrm{~d} \tau}\right)=\left[\frac{1}{y} \pm \frac{|H|}{\alpha}\right]^{1 / 2}
$$

with $\tau=(2 \alpha)^{1 / 2} t$ as the normalized time. We assume that $\alpha$ is positive, and take $y(0)=1$ to be the initial value, such that

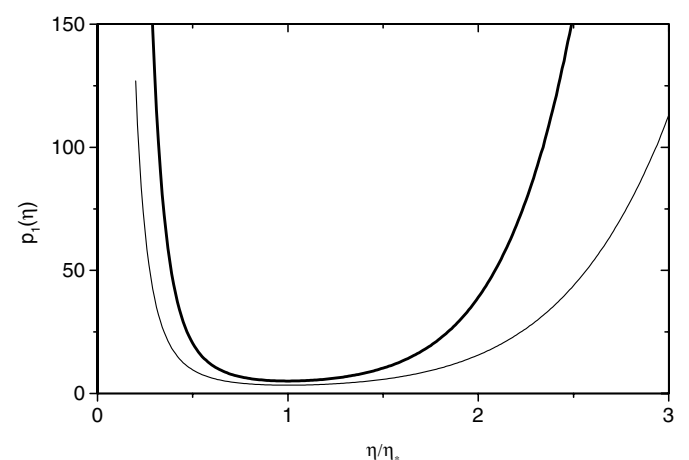

Fig. 9. The explicit radial dependence of pressure profile $\bar{p}_{1}(\eta)$ is shown as a function of the normalized radial label $\eta / \eta_{*}$ through the thick line. The explicit radial dependence of mass density profile $\bar{\rho}_{1}(\eta)$ is also shown through the thin line for comparisons.

the Lagrangian label $\eta$ corresponds to the initial position of the Eulerian fluid element. Equation (33) indicates an asymptotically convergent solution, with $H=-|H|<0$ negative, which is shown in the lower curve of Fig. 8 with $|H| / \alpha=0.5$. This track represents an asymptotically stable radius for the equatorial plasma torus. On the other hand, if the energy density $H=+|H|>0$ is positive, there is an ever expanding evolution track with a terminal velocity $\mathrm{d} y / \mathrm{d} \tau=(H / \alpha)^{1 / 2}$. This is shown in the upper curve of Fig. 8, which is inappropriate for AGB slow winds.

To better understand the amplitude and the sign of $H$, we multiply Eq. (30a) by $2 \rho \eta \mathrm{d} \eta$, and integrate to derive

$2 H \int \rho \eta \mathrm{d} \eta=\left(\frac{\mathrm{d} y}{\mathrm{~d} t}\right)^{2} \int \rho \eta \mathrm{d} \eta-2 \int\left(\frac{\partial p}{\partial r}+\rho \frac{G M}{r^{2}}\right) \mathrm{d} r$.

The first integral on the right side is positive definite, and corresponds to the kinetic energy of the expanding structure. Inside the second integral, there is a plasma pressure term, and a gravitational term. The gravitational term is always positive. Within the plasma pressure term, we have $\partial \bar{p}_{1}(\eta) / \partial \eta<0$ for $\eta<\eta_{*}$, since $\bar{p}_{1}(\eta)$ has a similar profile as $\bar{\rho}_{1}(\eta)$, according to Eq. (14a). This part has a forward plasma pressure force. For $\eta>\eta_{*}$, we have $\partial \bar{p}_{1}(\eta) / \partial \eta>0$, and the corresponding plasma pressure force is backward. It is clear that the explicit part of the pressure profile $\bar{p}_{1}(\eta)$, which is described by the explicit part of the mass density profile $\bar{\rho}_{1}(\eta)$ in Eq. (14a), contributes to the sign of $H$. Rewriting Eq. (20a) as

$\bar{\rho}_{1}^{1 / 3}(\eta)=\frac{1}{4 \alpha_{2}} \frac{\rho_{0}}{p_{0}} \alpha \eta_{*}^{2}\left[\frac{1}{2}\left(\frac{\eta}{\eta_{*}}\right)^{2}+\left(\frac{\eta_{*}}{\eta}\right)\right]$,

and assuming that the coefficient on the right side has a value of unity, these two profiles are shown in Fig. 9, where we have taken $\eta=3 \eta_{*}$ or $z=3 z_{*}=6$ as the upper bound in Fig. 9, as an example. The pressure profile in the positive gradient region $\eta>\eta_{*}$ helps to cancel the effects of the negative gradient, over the region $\eta<\eta_{*}$, in the above equation. Assuming that the slow wind velocity is below the escape velocity, the first integral on the right side is less than the gravitational term of the second integral. This implies that $H$ is negative, due to the overall negative sign on the second integral. The plasma torus is, therefore, asymptotically stationary.

When deriving the full radial profile of the plasma pressure, we caution that Fig. 9 is only the explicit part, $\bar{p}_{1}(\eta)$, of the radial profile relevant to Eq. (34). Another radial contribution comes from the implicit part, $\bar{p}_{2}(\bar{P})$. The full radial pressure profile is a combination of the explicit and the implicit part, 


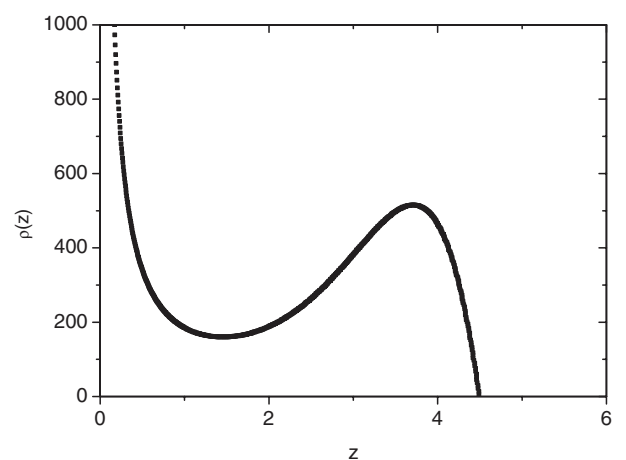

Fig. 10. The full, explicit and implicit, radial mass density profile $\bar{\rho}(\eta, \bar{P})$ using the homogeneous solution $R_{0}(z)$ is shown as a function of $z$.

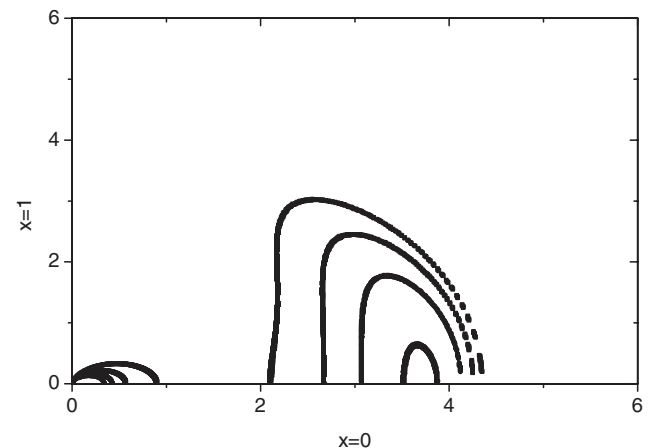

Fig. 11. The mass density contours $\bar{\rho}(\eta, \bar{P})$ with $\bar{C}=0$ are shown with $C=+200, C=+300, C=+400$, and $C=+500$ from the outer to inner contours respectively.

$\bar{p}(\eta, \bar{P})=p_{0} \bar{p}_{1}(\eta) \bar{p}_{2}(\bar{P})$, where $p_{2}(\bar{P})=\bar{P}(\eta, x)=R(\eta) \Theta(x)$. Since $\bar{p}_{1}(\eta)=\bar{\rho}_{1}^{\gamma}(\eta)$ by Eq. (14a) and $\bar{p}_{2}(\bar{P})=\alpha_{2} \bar{\rho}_{2}(\bar{P})$ by Eq. (20b), we could plot the full mass density profile, which reflects the plasma pressure profile with $\bar{C}=0$, as

$$
\begin{aligned}
\bar{\rho}(\eta, \bar{P}) & =\rho_{0} \bar{\rho}_{1}(\eta) \bar{\rho}_{2}(\bar{P}) \\
& =\rho_{0}\left[\frac{1}{4 \alpha_{2}} \frac{\rho_{0}}{p_{0}} \frac{\alpha z_{*}^{2}}{a^{2}}\right]^{3}\left[\frac{1}{2}\left(\frac{z}{z_{*}}\right)^{2}+\left(\frac{z_{*}}{z}\right)\right]^{3} \frac{1}{\alpha_{2}} R(\eta) \Theta(x) .
\end{aligned}
$$

With $z_{*}=2$ and considering $R(\eta)=R_{0}(\eta)$, the full radial dependence $\bar{\rho}_{1}(\eta) R_{0}(\eta)$, with the value of the coefficient in Eq. (36) taken to be unity, is shown in Fig. 10 for the plasma torus with a very pronounced maximum at $z=3.7$, and zero at $z=4.5$. The corresponding mass density spatial contours of $\bar{\rho}(\eta, \bar{P})$ are shown in Fig. 11.

\section{Bipolar planetary nebulae}

In addition to the equatorial plasma torus, our self-similar MHD formulation is able to model planetary nebulae of the bipolar type. For this purpose, we consider

$\bar{Q}=0$,

to warrant $B_{\phi}=0$. Following the same analysis, the radial function becomes

$R(\eta)=R_{0}(\eta)+R_{1}(\eta)=A_{0} z^{-1}-a_{12} z^{12}-a_{9} z^{9}-a_{6} z^{6}-a_{3} z^{3}+a_{0}$.

The coefficients are $(12 \times 11-2) a_{12}=A^{(12)}=A,(9 \times 8-2) a_{9}=$ $A^{(9)}=4 \kappa^{3} A,(6 \times 5-2) a_{6}=A^{(6)}=6 \kappa^{6} A,(3 \times 2-2) a_{3}=A^{(3)}=$ $4 \kappa^{9} A$, and $2 a_{0}=A^{(0)}=\kappa^{12} A$. Although we have continued to

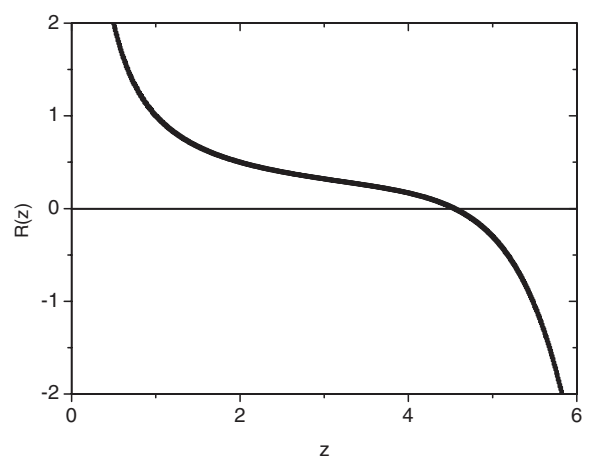

Fig. 12. The general solution of the radial function $R(z)$ is shown as a function of $z$.

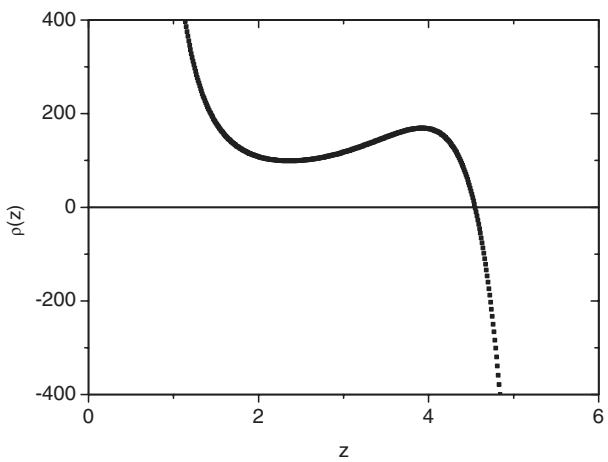

Fig. 13. The full, explicit and implicit, radial mass density profile $\bar{\rho}(\eta, \bar{P})$ is shown as a function of $z$.

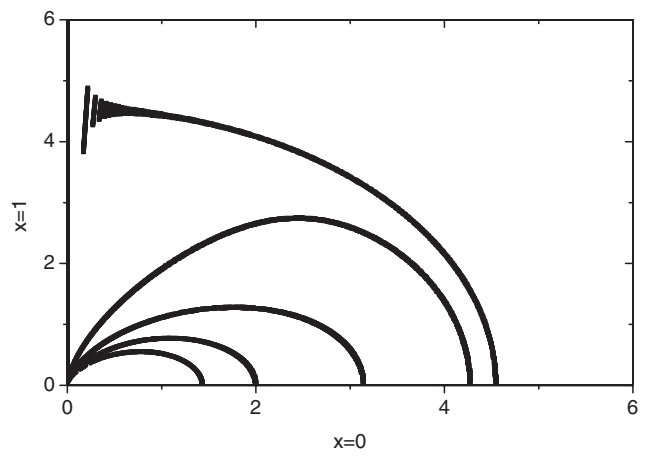

Fig. 14. The magnetic field lines are shown with $C=+0.0, C=+0.1$, $C=+0.3, C=+0.5$, and $C=+0.7$ from the outer to inner contours respectively.

use $z=a \eta$ as the normalized radial label, the normalization factor $a$ here has no connection to $\bar{Q}$ because we now assume that $\bar{Q}=0$. We could imagine that $a=k$, such that $z=k \eta$. We notice that the particular solution consists entirely of negative terms, apart from the final term, whereas the homogeneous solution is positive. With $A_{0}=1, A=10^{-7}, z_{*}=2, \kappa^{3}=2 z_{*}^{3}=16$, the general solution $R(\eta)$ shown in Fig. 12 becomes negative at $z=z_{0}=4.55$. This value of $z_{0}$ happens to be close to the roots of $R_{0}(z)$ and $R_{1}(z)$, of the equatorial plasma torus case, where $\bar{Q}=a \bar{P} \neq 0$. This fact is purely a coincidence. The full radial profile of the plasma density, described by Eq. (36), explicit and implicit parts, is shown in Fig. 13. Since plasma density is positive definite, $z \leq z_{0}$ defines the physical domain of our selfsimilar solution. The bipolar field contours are shown in Fig. 14. The radial component vanishes at $z_{0}, B_{r}\left(z_{0}\right)=0$, which is the boundary of the expanding bipolar structure. 
To conclude, we have represented the AGB wind by an exploding ideal MHD plasma. The explosion is modelled by an isotropic radial plasma velocity in spherical coordinates. Through dissipation, this exploding plasma is believed to undergo self-organization. The possible self-organized states may or may not be spherically symmetric, although the velocity will be spherical symmetric. Self-organization implies that the plasma global conservation properties are sufficiently dominant to drive the system through dissipation, independently of the initial conditions. To find the possible self-organized states, we solve the ideal MHD equations, with $\gamma=4 / 3$, for self-similar solutions in time, where the temporal and spatial dependences of each physical variable are in separable form. We identify these self-similar solutions of ideal MHD to be the self-organized configurations reached by dissipation. We would like to emphasize the word ideal, which means that this process occurs without dissipation. Although self-organized states are physically generated by dissipation, they are part of the ideal MHD solutions. Dissipation provides the means by which an ideal state, of a particular energy density, is topologically transformed to another ideal state, of lower energy density. Using this time self-similar formulation, we can derive possible end states without time iteration, assuming a specified initial configuration. By circumventing intermediate stages, this self-similar approach, which is assumed to be independent of self-organization physical arguments, appears to be developing the required solutions for the problems waiting to be solved.

Under axisymmetry, we have established two solutions for equatorial ejection. The first solution has a finite azimuthal magnetic field, and represents a plasma torus with closed toroidal field lines. Beyond the plasma torus, the magnetic field lines are open and the plasma is not confined. This plasma torus provides the principle structure for the interacting-wind model. The second solution has a null azimuthal field, and represents a bipolar planetary nebula. Whether these self-organized structures continue to expand indefinitely depends on the integration constant $H$ of the evolution function, which could have an asymptotically bounded track for a stable torus, and an unbounded track for an ever expanding bipolar nebulae. Additional nebula features, such as filaments and jets, could be accounted for with a fully three-dimensional self-similar MHD model.

We in fact believe that this description of self-organized plasmas, using self-similar solutions, could represent a fundamental process in astrophysical ejection events. Apart from the equatorial ejection solutions of plasma torus and bipolar structure of planetary nebulae discussed here, the highly collimated polar ejection solutions could be relevant to extragalactic jets, should we consider these objects to be ejection events (Tsui \& Serbeto 2007). The collimated polar ejection mechanism could also be relevant to some asymmetric supernovae, with recoil on the neutron star, and to quasar ejection models of active galactic nucleus in cosmology.

Acknowledgements. The author is deeply grateful to Prof. Akira Hasegawa for the very essential concept of self-organization in fluids and plasmas.

\section{References}

Balick, B., Rugers, M., Terzian, Y., \& Ghengalur, J. N. 1993, ApJ, 411, 778 Bian, F. Y., \& Lou, Y. Q. 2005, MNRAS, 363, 1315

Biskamp, D. 1993, Nonlinear Magnetohydrodynamics (Cambridge: Cambridge University Press), Chap. 2

Blandford, R. D., \& Payne, D. G. 1982, MNRAS, 199, 883

Bogovalov, S., \& Tsinganos, K. 1999, MNRAS, 305, 211

Chevalier, R. A., \& Luo, D. 1994, ApJ, 421, 225

Dyson, J. E., \& de Vries, J. 1972, A\&A, 20, 223

Gardiner, T. A., \& Frank, A. 2001, ApJ, 557, 250

Garcia-Segura, G. 1997, ApJ, 489, L189

Hasegawa, A. 1985, Adv. Phys., 34, 1

Jordan, S., Werner, K., \& Ot'Toole, S. J. 2005, A\&A, 432, 273

Kahn, F. D., \& West, K. A. 1985, MNRAS, 212, 837

Kwok, S., Purton, C. R., \& FitzGerald, P. M. 1978, ApJ, 219, L125

Landau, L. D., \& Lifshitz, E. M. 1978, Fluid Mechanics (Oxford: Pergamon Press), Chap. II

Lopez, J. A., Meaburn, J., \& Palmer, J. W. 1993, ApJ, 415, L135

Lou, Y. Q., \& Shen, Y. 2004, MNRAS, 348, 717

Lou, Y. Q., \& Gao, Y. 2006, MNRAS, 373, 1610

Lou, Y. Q., \& Wang, W. G. 2006, MNRAS, 372, 885

Lou, Y. Q., \& Wang, W. G. 2007 [arXiv:0704.0223v1]

Low, B. C. 1982a, ApJ, 254, 796

Low, B. C. 1982b, ApJ, 261, 351

Low, B. C. $1984 a$, ApJ, 281, 381

Low, B. C. 1984b, ApJ, 281, 392

Matt, S., Balick, B., Winglee, R., \& Goodson, A. 2000, ApJ, 545, 965

Mellema, G., Eulderink, F., \& Icke, V. 1991, A\&A, 252, 718

Miranda, L. F., \& Solf, J. 1992, A\&A, 260, 397

Osherovich, V. A., Farrugia, C. J., \& Burlaga, L. F. 1993, J. Geophys. Res., 98, 13225

Osherovich, V. A., Farrugia, C. J., \& Burlaga, L. F. 1995, J. Geophys. Res., 100, 12307

Pascoli, G. 1993, J. Astrophys. Astr., 14, 65

Sommerfeld, A. 1950, Mechanics of Deformable Bodies (New York: Academic Press), Chap. III

Tsui, K. H. 2006, Phys. Plasmas, 13, 072102

Tsui, K. H., \& Tavares, M. D. 2005, J. Atmos. Solar-Terr. Phys., 67, 1691

Tsui, K. H., \& Serbeto, A. 2007, ApJ, 658, 794

Tsui, K. H., Navia, C. E., Robba, M. B., Carneiro, L. T., \& Emelin, S. E. 2006, Phys. Plasmas, 13, 113503

Yu, C., \& Lou, Y. Q. 2005, MNRAS, 364, 1168 